\title{
Técnica de ex vivo en modelo biológico para trasplante pulmonar. Una manera de realizar simulación de alto realismo
}

\section{Ex vivo technique in a biological model for lung transplantation. A way to perform high realism simulation}

\author{
Juan Carlos Vázquez-Minero, ${ }^{*}$ Claudia Hernández-Jiménez,* Mariana Silva-Martínez,* \\ Javier Martínez-Cortés, ${ }^{*}$ Juan Raúl Olmos-Zúñiga, ${ }^{*}$ Matilde Baltazares-Lipp,* María Teresa Pérez-López, \\ Emilio Roberto Rueda-Juárez,* Rogelio Jasso-Victoria*
}

*Instituto Nacional de Enfermedades Respiratorias Ismael Cosío Villegas, Ciudad de México.

\begin{abstract}
RESUMEN. El trasplante pulmonar es un tratamiento que implica el dominio de una técnica quirúrgica compleja para el especialista en formación en cirugía de tórax. Una modalidad de preservación pulmonar antes de ser trasplantado es la técnica de ex vivo. Material y métodos: Para capacitar al residente en esta área se utilizó un modelo biológico de cerdo para hacer la obtención del bloque corazón-pulmón y su conexión al sistema ex vivo. Resultados: Se realizaron 20 procuraciones del bloque corazón-pulmón y se conectaron al sistema ex vivo sin complicaciones, dejándose conectados por dos horas para valorar que la técnica fuera la correcta. Esta modalidad de aprendizaje significativo con un proceso de cognición situada ayuda a mejorar el aprendizaje de los residentes en formación. Conclusiones: La simulación biológica de alto realismo es una herramienta muy útil para el aprendizaje significativo y situado de los residentes de cirugía torácica.
\end{abstract}

Palabras clave: Simulación, modelo biológico, técnica ex vivo.

\section{INTRODUCCIÓN}

El trasplante pulmonar es la alternativa más viable en nuestro tiempo para resolver la patología pulmonar terminal. En los últimos años se ha incrementado el número de trasplantes realizados según la International Society Heart and Lung Trasplantation alcanzando para 2013 un total de 3,719.

También la sobrevida ha experimentado una mejoría a uno y cinco años, siendo en 1994 de 72 y 49\%, alcanzando en el año 201380 y 54\%, respectivamente. ${ }^{2}$ Lo anterior se debe a muchas causas, entre las que se cuentan aquéllas que presentan mejores soluciones de preservación del

Correspondencia:

Dr. Juan Carlos Vázquez Minero

Instituto Nacional de Enfermedades Respiratorias Ismael Cosío

Villegas, Ciudad de México.

Correo electrónico: minerojc@hotmail.com

Trabajo recibido: 30-VII-2019; aceptado: 08-V-2020.
ABSTRACT. Pulmonary transplantation is a treatment that involves mastery of a complex surgical technique, for the specialist in training in chest surgery. One modality of preserving the lung before being transplanted is the ex vivo technique. Material and methods: In order to train the resident in this area, a biological model of pig was used to obtain the heart lung block and its connection to the living system. Results: 20 lung heart block procurements were carried out and connected to the lively system without complications, leaving them connected for two hours to assess, that the technique was correct. This significant learning modality, with a process of situated cognition, helps to improve the learning of residents in training. Conclusions: High-realism biological simulation is a very useful tool for meaningful and situated learning of thoracic surgery residents.

Keywords: Simulation, biological model, ex vivo technique.

órgano, una mejor técnica de anastomosis bronquial, la introducción de inmunosupresores más selectivos, los criterios de selección tanto del donante como del receptor y el aporte de las técnicas de circulación extracorpórea cuando hay una disfunción del injerto.,

Los hospitales donde se realiza la atención de pacientes con patología pulmonar como el Instituto Nacional de Enfermedades Respiratorias (INER) Ismael Cosío Villegas, deben tener necesariamente un dominio adecuado de las técnicas de trasplante pulmonar y de las nuevas alternativas de preservación del injerto. Una de estas técnicas es el procedimiento de ex vivo.

Esta técnica permite hacer funcionar a los pulmones fuera del cuerpo humano con condiciones fisiológicas a una temperatura de $37^{\circ}$ con circulación y ventilación y con condiciones metabólicas normales, además permite a través de ella evaluar, recuperar y optimizar los pulmones para su posterior trasplante. ${ }^{5}$

La simulación en la educación médica ha presentado un desarrollo importante a nivel mundial, ésta se ha con- 
vertido en una herramienta mediante la cual se favorece la adquisición de habilidades clínicas previo al contacto real con el paciente y fomenta la seguridad para éste mediante destrezas para disminuir la posibilidad de errores o complicaciones en la realización de procedimientos. Existen varios tipos de simulación, el más complejo es con modelos biológicos que presenta un alto realismo. ${ }^{6}$

Es de vital importancia como grupo médico encargado de la atención, conocer y dominar esta técnica de perfusión. Por tanto, para el aprendizaje de los médicos residentes en formación se hizo una serie de escenarios de simulación, utilizando un modelo biológico de alto realismo con cerdos para llevar a cabo la procuración y perfusión ex vivo y así poder practicar las técnicas y reforzar los conocimientos sin riesgo para los pacientes y en un ambiente seguro para los residentes.

\section{MATERIAL Y MÉTODOS}

\section{Escenarios de simulación}

Se realizaron 20 escenarios de simulación biológica de alto realismo con la procuración del bloque corazón-pulmón. Se utilizaron 20 cerdos Landrace clínicamente sanos, sin importar el sexo, con un peso entre $20-22 \mathrm{~kg}$, los cuales fueron obtenidos del Área del Bioterio del INER Ismael Cosío Villegas. Este protocolo fue aprobado por el Comité de Bioética del Instituto (número de protocolo B09-17) y se realizó de acuerdo con las especificaciones técnicas para el cuidado y uso de animales de laboratorio de la Norma Oficial Mexicana (Estados Unidos Mexicanos. AFÍA. Especificaciones Técnicas para la Producción, Cuidado y Uso de Animales de Laboratorio de la Norma Oficial Mexicana NOM-062ZOO-1999. Diario Oficial de la Federación 06 diciembre, 1999) y la Guide for the Care and Use of Laboratory Animals of USA (National Institutes of Health USA, Guía para el Cuidado y Uso de los Animales de Laboratorio. Department of Health and Human Services, Public Health Service, National Institutes of Health USA. Edición Mexicana Auspiciada por la Academia Nacional de Medicina. México, D.F. 2002). ${ }^{7,8}$

Los escenarios consistieron en:

1. Con el espécimen bajo anestesia general endovenosa y ventilación mecánica asistida con estabilidad hemodinámica se realizó la procuración del bloque corazónpulmón, haciéndose la preparación para la conexión al sistema de ex vivo. Ésta fue efectuada por un residente de cuarto o tercer año de cirugía de tórax y un residente de segundo año (Figura 1).

2. Se conecta el bloque preparado al sistema de ex vivo y se evalúa su adecuado funcionamiento (Figura 2). Esta conexión al sistema de ex vivo es auxiliada por el personal de investigación y perfusionista.
3. Se deja funcionando el sistema y se evalúan fugas de la vía aérea y datos de fuga del sistema vascular durante dos horas (Figura 3).

4. Se da por terminada la simulación al cumplir dos horas de perfusión, posteriores a las cuales se continúa el protocolo de ex vivo por parte del equipo de cirugía experimental, acorde a lo planeado por ellos.

5. El equipo de residentes y del área de investigación y perfusión realizan un análisis de lo que hicieron, los puntos a favor y en contra así como las áreas de oportunidad.

\section{RESULTADOS}

Se llevaron a cabo las 20 simulaciones de ex vivo, con un tiempo de una hora para la procuración y 30 minutos para la conexión al sistema de ex vivo. No se presentaron complicaciones con procuración de bloque corazón-pulmón y conexión al sistema ex vivo dejando que se realizara la perfusión y ventilación por dos horas, sin datos de fuga de la vía aérea ni del sistema vascular. Dándose por terminado el proceso para los fines de este estudio.

La simulación fue realizada por residentes de segundo a cuarto año de cirugía de tórax donde se puso en práctica la disección y separación del bloque corazón-pulmón, sin presentarse lesiones vasculares o de vía aérea. La conexión del sistema ex vivo también fue efectuada por residentes de segundo a cuarto año de cirugía y asistencia del personal de investigación y perfusión, conectando a ventilación y perfusión al injerto para fines del este estudio por 30 minutos.

La manera de evaluar los escenarios fue a través de observar la adecuada función del bloque ya conectado al sistema ex vivo sin presentar fuga aérea o salida del líquido de perfusión por el sistema vascular por un período de dos

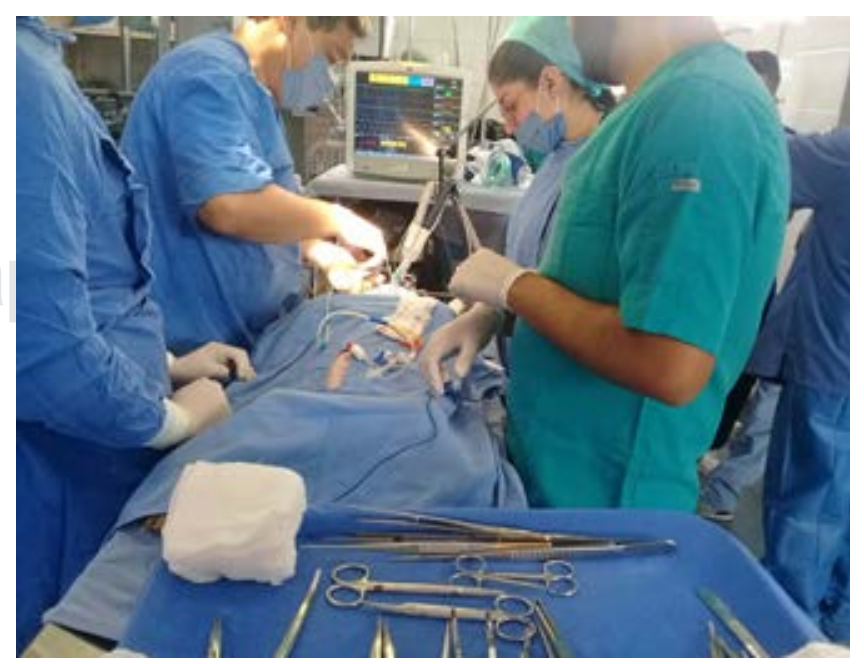

Figura 1: Equipo quirúrgico procurando bloque corazón-pulmón. 


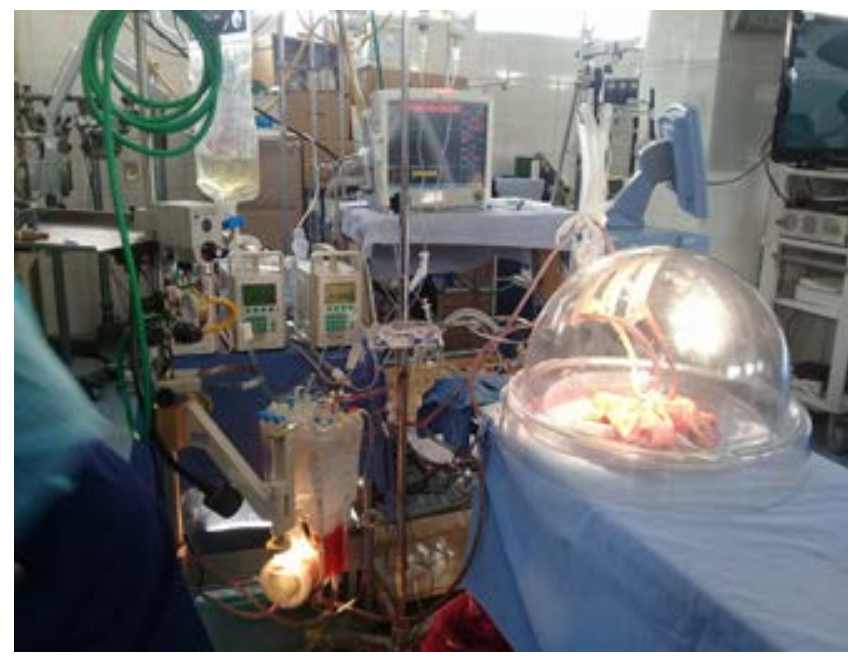

Figura 2: Sistema ex vivo funcionando con bloque pulmonar.

horas. Al final cada grupo hizo una reflexión acerca de los aciertos y errores durante el proceso. Los residentes concluyeron que les fue de mucha utilidad para el aprendizaje y reforzamiento de la técnica de procuración del bloque corazón-pulmón y el sistema ex vivo.

\section{DISCUSIÓN}

Una de las técnicas quirúrgicas más complejas a las que un cirujano torácico se va a enfrentar a lo largo de su vida profesional es el trasplante pulmonar, que consiste en transferir un pulmón o ambos pulmones de un individuo (donador) a otro (receptor), con el objeto de reemplazar la función de los que se encuentran dañados. Bajo estas aristas es muy importante que el residente desde su formación se familiarice con estas técnicas, mismas que en nuestro país no son frecuentes. ${ }^{9}$

En la actualidad una modalidad de preservación del órgano es la técnica de ex vivo descrita antes. Dentro del entrenamiento del cirujano es necesaria la familiarización con esta técnica, una manera segura de enseñarla es a través de la simulación con un modelo biológico que permita hacer el procedimiento de manera segura e ir corrigiendo defectos durante su realización. Para enseñar a un médico y en especial a un cirujano deben existir recursos que permitan al alumno tener prácticas lo más cercanas a la realidad y evitar realizarlas en pacientes para no causarles daños innecesarios. Es bajo esta circunstancia donde la simulación en medicina y en particular en cirugía adquiere un papel importante..$^{10}$

El paradigma de la cognición situada representa una de las tendencias actuales más representativas y promisorias de la teoría y la actividad sociocultural. La cirugía se aprende de persona a persona, de un experto a un aprendiz. La simulación biológica de alto realismo permite que el alumno se enfrente a un escenario que puede presentar todas las complicaciones y situaciones de un escenario real y que con esto pueda llevar a cabo técnicas quirúrgicas adecuadas para resolver estos problemas. Un grupo de residentes realiza la práctica con diferentes grados de conocimiento y adiestramiento. ${ }^{11}$

La simulación es una herramienta que se utiliza en el aprendizaje significativo dentro del grupo de ejercicios, demostraciones y simulaciones situadas. En nuestro caso ponemos al alumno ante una situación compleja y real que es la técnica de ex vivo, donde pudo practicar la procuración del bloque corazón-pulmón y conectarlo al sistema artificial de preservación del órgano. ${ }^{12}$

Al presente, la enseñanza de la cirugía se ha visto afectada por muchos factores, el más importante es la seguridad del paciente, en quien los procedimientos no deben implicar un riesgo, incluso en cirugías poco comunes o poco frecuentes como el trasplante. En este momento la simulación desempeña un papel preponderante que permite el entrenamiento de las distintas habilidades que componen el espectro de la competencia profesional. Así es como los residentes tienen la oportunidad de hacer la procuración del bloque y la conexión a un sistema de mantenimiento del órgano. ${ }^{13}$

La simulación por sus características intrínsecas es un método idóneo para el entrenamiento de equipos: permite practicar la comunicación, la toma de decisiones y el manejo de recursos en situaciones de crisis. Todos estos puntos fueron puestos en práctica por los residentes en estos escenarios de simulación biológica, cada uno con diferencias de los otros. ${ }^{14}$

La simulación estimula el aprendizaje a partir de un ambiente emocionante y el recuerdo de la experiencia. Esto se explica en el modelo del afecto, es decir, de cómo el alumno integra esta experiencia a su práctica al ser totalmente significativa. Los residentes al hacer una

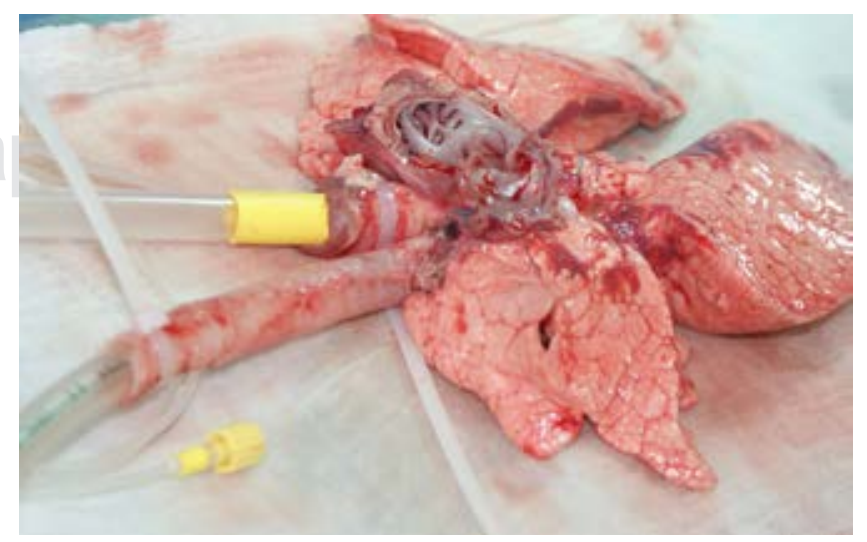

Figura 3: Cánulas funcionando sin fugas. 
reflexión de lo hecho, con aciertos y errores, reforzaron o adquirieron conocimientos que por este proceso van a ser más duraderos. ${ }^{15,16}$

Para que un alumno pueda tener un adecuado razonamiento durante la práctica de un escenario simulado, es necesario que tenga dos dimensiones: una cultural, es decir, que el ejemplo sea relevante dentro del campo de acción del alumno; y el segundo, de actividad social, la cual hace que exista un aprendizaje colaborativo que al final le sea útil en su vida futura. Por ello decidimos que los escenarios fueran complejos enfocados al trasplante y ex vivo y, además, en equipo para hacerlo colaborativo y sociocultural. ${ }^{17}$

Al final, mencionaremos que la simulación de alto realismo con modelo biológico en los residentes acortó esa brecha educacional entre «ver uno» $y$ «hacer uno» en estos procedimientos complejos. ${ }^{18}$

\section{CONCLUSIONES}

La simulación biológica de alto realismo es una herramienta muy útil para el aprendizaje significativo y situado de los residentes de cirugía torácica, sobre todo en aquellos procedimientos que por su complejidad no deben ni pueden ser probados en los pacientes.

\section{REFERENCIAS}

1. Yusen RD, Edwards LB, Kucheryavaya AY, et al.; International Society for Heart and Lung Transplantation. The Registry of the International Society for Heart and lung transplantation: thirty-first adult lung and heart-lung transplant report-2014; Focus Theme: Retransplantation. J Heart Lung Transplant 2014;33(10):1009-1014. https://doi.org/10.1016/j.healun.2014.08.004

2. Christie JD, Edwards LB, Kucheryavaya AY, et al. The Registry of the International Society for Heart and lung transplantation: twentyeighth adult lung and heart-lung transplant Report-2011. J Heart Lung Transplant 2011;30(10):1104-1122. https://doi.org/10.1016/j. healun.2011.08.004.

3. Murthy SC, Gildea TR, Machuzak MS. Anastomotic airway complications after lung transplantation. Curr Opin OrganTransplant 2010;15(5):582-587. https://doi.org/10.1097/MOT.0b013e32833e3e6e

4. Currey J, Pilcher DV, Davies A, et al. Implementation of a management guideline aimed at minimizing the severity of primary graft dysfunction after lung transplant. J Thorac Cardiovasc Surg 2010;139(1):154-161. https://doi.org/10.1016/j.jtcvs.2009.08.031

5. Moradiellos DF, Varela UA. Perfusión ex vivo: evaluar, recuperar y optimizar pulmones humanos para trasplante. Arch Bronconeumol 2010;46(5):213-214. https://doi.org/10.1016/j.arbres.2010.02.001.

6. Dávila CA. Simulación en educación médica. Inv Ed Med 2014;3(10):100-105.

7. Especificaciones Técnicas para la Producción, Cuidado y Uso de Animales de Laboratorio de la Norma Oficial Mexicana NOM-062ZOO-1999. Diario Oficial de la Federación, 6 de diciembre, 1999. Estados Unidos Mexicanos.

8. Guía Para el Cuidado y Uso de Los Animales de Laboratorio. Department o Health and Human Services, Public Health Service, National Institutes of Health U.S.A. Edición Mexicana Auspiciada por la Academia Nacional de Medicina. México, D.F., 2002.

9. Montero R, Vicente R. Tratado de trasplantes de órganos. Tomo II. Madrid: ARAN Publicaciones Médicas; 2006.

10. Iñiguez GM, Jasso VR, Olmos ZR, et al. Trasplante pulmonar en un modelo experimental. Capacitación para residentes de cirugía torácica. Neumol Cir Torax 2013;72(1):25-31.

11. Daniels H. Vigotsky y la pedagogía. Barcelona: Paidós; 2006.

12. Díaz-Barriga AF. Cognición situada y estrategias para el aprendizaje significativo. Revista Electrónica de Investigación Educativa 2003:5(2):105-117. http://redie.ens.uabc.mx/vol5no2/contenido-arceo. html.

13. Ruiz GJ, Martín PJ, González NM, Redondo FC, Manuel PJ. La simulación como modelo de enseñanza en cirugía. Cir Esp 2018;96(1):12-17. doi: 10.1016/.ciresp.2017.09.005.

14. Aggarwal R, Darzi A. Innovation in surgical education-- $A$ driver for change. Surgeon 2011;9 Suppl 1:S30-S31. https://doi.org/10.1016/j. surge.2010.11.021.

15. Posner J, Rusell JA, Petreson BS. The circunflex model of affect: An integrative approach to affective neuroscience, cognitive development, and pshysicopathologic. Dev Psychopatol 2005;17(3):715-734. https:// doi.org/10.1017/s0954579405050340.

16. Feldman-Barret L, Rusell J. Cicunflex model off affect. The Oxford companion to emotion and the affective. Am Psychol Soc 1999;8:10-14.

17. Derry S, Levin J, Schauble L. Stimulating statistical thinking though situated simulations. Teaching of Psychology 1995;22(1):51-57.

18. Akaike M, Fukutomi M, Nagamune M, et al. Simulation-based medical education in clinical skill laboratory. J Med Invest 2012;59(1-2):28-35. https://doi.org/10.2152/jmi.59.28

Conflicto de intereses: Los autores declaran no tener conflicto de intereses. 